\title{
Dipolar Rotors Orderly Aligned in Mesoporous Fluoro- organosilica Architectures
}

\author{
S. Bracco, ${ }^{1}$ M. Beretta, ${ }^{1}$ A. Cattaneo,${ }^{1}$ A. Comotti, ${ }^{1 *}$ A. Falqui, ${ }^{2}$ K. Zhao,${ }^{3}$ C. Rogers,${ }^{3}$ P. Sozzani ${ }^{1 *}$
}

\begin{abstract}
New mesoporous covalent frameworks, based on hybrid fluoro-organosilicas, were prepared to realize a periodic architecture of fast molecular rotors containing dynamic dipoles in their structure. The mobile elements, designed on the basis of fluorinated $p$ divinylbenzene moieties, were integrated into the robust covalent structure through siloxane bonds, and showed not only the rapid dynamics of the aromatic rings (ca. $10^{8} \mathrm{~Hz}$ at $325 \mathrm{~K}$ ), as detected by solid-state NMR, but also a dielectric response typical of a fast dipole reorientation under the stimuli of an applied electric field. Furthermore, the mesochannels are open and accessible to diffusing-in gas molecules, and rotor mobility could be individually regulated by $\mathrm{I}_{2}$ vapors. The iodine enters the channels of the periodic structure and reacts with the pivotal double bonds of the divinyl-fluoro-phenylene rotors, affecting their motion and the dielectric properties.
\end{abstract}

The fabrication of molecular rotors in solids is the objective of a challenging research activity that offers the fascinating perspective of molecular mobility, which could be a mediator between micro- and macroscopic phenomena. ${ }^{1}$ Indeed, a variety of applications have been envisaged: the fabrication of switchable ferroelectrics, sensors, and also materials endowed with optical properties. ${ }^{2}$ However, these goals will remain unrealized unless dipoles can be integrated into the rotors to create oscillating dipoles in the chemical structure. ${ }^{3}$ Thus, the construction of an ordered assembly of fast-rotating dipoles in solids offers the stimulating scope of realizing new functional properties, provided the dipolar rotors are engineered into robust materials in which they self-organize as arrays to form spatially-coherent architectures.

Materials containing dipolar molecular rotors are still uncommon and so far they have been realized by exploiting the gain in freedom provided by surfaces and by molecular crystals with frustrated packing, as well as by mobile guests included in suitable hosts. ${ }^{4}$ Starting from the consideration that robust covalent frameworks appear suitable for sustaining rotors, because they can provide stable support to the dynamic elements, we pursued the idea of integrating dipole-bearing rotors

[1] Dr. S. Bracco, Dr. M. Beretta, Dr. A. Cattaneo, Prof. A. Comotti, Prof. P. Sozzani

Department of Materials Science

University of Milano Bicocca

Via R. Cozzi 55, Milano, Italy

E-mail: piero.sozzani@mater.unimib.it

angiolina.comotti@mater.unimib.it

[2] Prof. A. Falqui

Biological and Environmental Sciences and Engineering Division King Abdullah University for Science and Technology

Thuwal 23955-6900, Kingdom of Saudi Arabia

[3] Dr. K. Zhao, Prof. C. Rogers

Department of Physics, University of Colorado Boulde

2000 Colorado Ave., Boulder, Colorado 80309 USA

Supporting information for this article is given via a link at the end of the document. in a covalent framework endowed with the beneficial property of porosity. The combination, in a single material, of rotating dipoles and porosity could produce novel properties derived from the accessibility of the dipolar rotors by the gas or vapor phases, thus modulating dynamics by the action of chemical species. To the best of our knowledge the preparation of dipolar rotors in porous frameworks has been reported in only a few cases of metal organic materials, ${ }^{5}$ while architectures constituted by covalent bonds have not yet been explored. The innovation brought by the present study is the insertion of dipole-bearing rotors in the structure of porous covalent materials.

This project required the design of molecular rotors with a non-centrosymmetric charge-distribution that produced a molecular dipole vector lying off the rotation axis, in such a way as to generate rotating dipole vectors. The choice of the polar groups mounted on rotors was dictated by the need to minimize the size and weight of the rotating elements in order to reduce, simultaneously, collisions with adjacent rotors and the moment of inertia of each rotor, both of which would hamper fast rotation. The smallest possible perturbation of the rotors could come from fluorine atoms, which produce C-F dipoles without substantial contribution to space occupancy. Therefore, we designed new porous frameworks in which carbon-fluorine dipoles undergo fast reorientation about the main axes of aligned $p$-phenyl molecular rotors on which they are mounted.

Periodic mesoporous organosilicas (PMOs) with crystal-like order in their walls were the materials of choice to sustain the dipolar rotors: they are robust covalent frameworks, stable in solvents and heat resistant, thus providing a valid platform to align the $p$-phenylene molecular rotors into ordered arrays. ${ }^{6}$ The sophisticated architecture with large open channels enables specialized functions and a facile accessibility of guests to organic active sites: in fact, PMOs have been proposed for light harvesting and conversion, as well as for vapor absorption and gas storage. ${ }^{7}$ However, fluorinated PMOs with crystalline order in their walls have not been reported to date.

Herein we present periodic mesoporous hybrid materials composed by fast-reorienting carbon-fluorine dipoles as well as their dynamics and absorptive behavior (Scheme 1). The materials are constituted by orderly arranged divinyl- $p$ fluorophenylene units bridging two adjacent siloxane layers, and

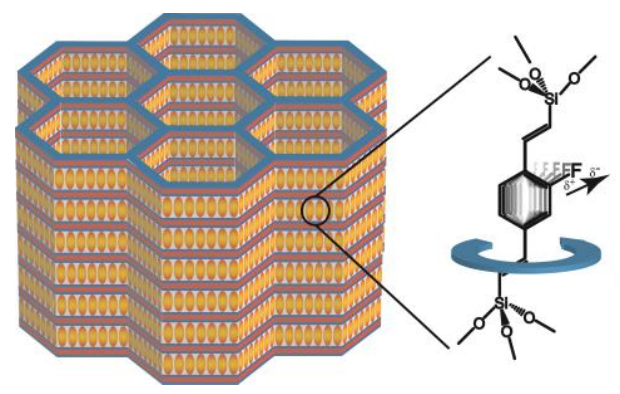


Scheme 1. Representation of the mesoporous organosilicas (left) and 2fluorodivinyl- and 2,3-difluoro-divinylbenzenesiloxane moieties (right). exhibit an intriguing periodic architecture that is hierarchically organized on both the molecular and the meso-scale. The rotating groups consist of dipolar monofluoro- and difluoro- $p$-phenylene moieties that supply a permanent electric molecular dipole moment and are aligned in the walls surrounding $4 \mathrm{~nm}$ wide nanochannels. The alignment of the rotation axes in the solid structure enabled the creation of collective phenomena through the exploitation of the fast rotating-dipole dynamics. Indeed, the high surface area developed by the materials, and the exposure of the divinyl moieties allowed reactions with guest species, such as molecular iodine, diffused from the vapor phase: this process can reduce the dipole reorientation reinforcing the prospect of tuning rotor motion by a chemical action.

The mesoporous 2-fluorodivinylbenzenesilica (FOS-1) and 2,3-difluorodivinylbenzenesilica (FOS-2) were fabricated by a template synthesis starting from the precursors $p$-bis $[(E)-2$ (triethoxylsilyl)vinyl]-2-fluorobenzene and $p$-bis[(E)-2(triethoxylsilyl)vinyl]-2,3-difluorobenzene that self-organize around the structural directing agent (octadecyltrimethylammoniumbromide) in sodium hydroxide aqueous solution at $\mathrm{PH}=13.75$, followed by the removal of the surfactant by an acidic solution in ethanol. Dipolar rotors were mounted on a covalent framework of siloxane bonds that keep the rotors aligned at a distance of $4.4 \AA$ from each other. The mesoporous fluoroorganosilicas exhibit a hierarchical order corresponding to hexagonal nanochannels and crystal-like regularity in the channel walls as shown by powder X-ray diffraction and TEM images (Figure 1). Indeed, powder X-ray diffraction of FOS-1 and FOS-2 showed, in the small-angle scattering region, a few typical peaks that revealed the $2 \mathrm{D}$ hexagonally ordered $\left(P_{6 \mathrm{~mm}}\right)$ mesopore arrangement with lattice constants of $51.8 \AA$ and $55.3 \AA$, respectively, while the lamellar ordering of the organic moieties within the pore walls was clearly demonstrated by the presence of signals at higher $2 \Theta$ angles $(d=12.0 / 6.0 / 4.0 / 3.0 \AA)$. The highly ordered organization of the structure was confirmed by High Resolution TEM (HRTEM) images and the resultant two dimensional filtered Fast Fourier Transform (2D-FFT) patterns (Figure 1). In the filtered 2D-FFT pattern of FOS-1 compound, spots due to the hexagonal arrangement on the mesoscale are apparent. Based on the data above, we can depict an idealized model for the compounds consisting of divinyl-2-fluoro- $p$ phenylene and divinyl-2,3-difluoro-p-phenylene moieties arranged, in an orderly fashion, in the walls, their main molecular axis being parallel to the channel direction and anchored at each end by a siloxane layer.

Nitrogen physisorption isotherms at $77 \mathrm{~K}$ demonstrated the open porosity of the fluorinated compounds: type-IV isotherms corresponding to uniform mesopores with Brunauer-Emmett-Teller (BET) specific surface areas of 860 and $704 \mathrm{~m}^{2} / \mathrm{g}$ and pore diameters of 33.4 and $36.0 \AA$ (BJH) were observed for FOS-1 and FOS-2, respectively (Figure 1a and Supporting Information). Carbon dioxide is also captured efficiently: the $\mathrm{CO}_{2}$ isotherms at $195 \mathrm{~K}$ and up to 1 bar showed a type-IV pattern confirming the mesoporous structure, and the remarkable uptake of $405 \mathrm{~cm}^{3}(\mathrm{STP}) / \mathrm{g}$ corresponding to $80 \%$ of $\mathrm{CO}_{2}$ by weight. The two fluorinated materials exhibited elevated thermal stability up to $500^{\circ} \mathrm{C}$, as disclosed by TGA analysis (Supporting Information).

a)

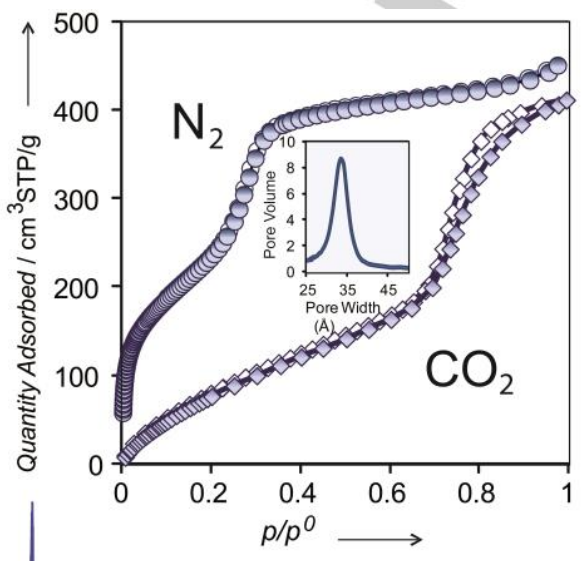

b)

c)
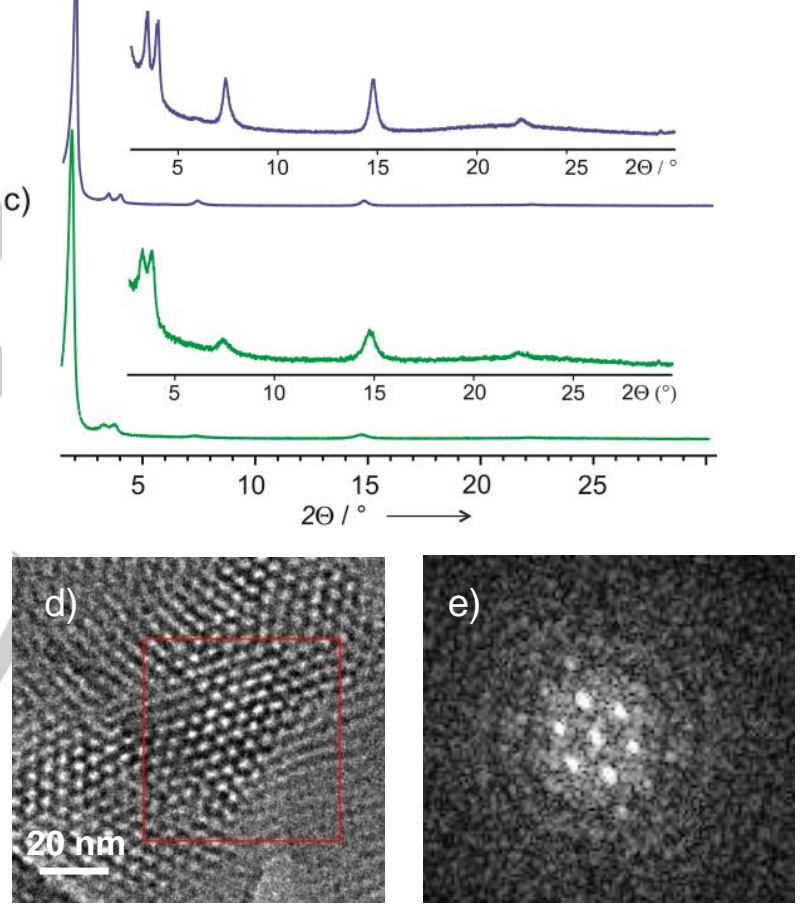

Figure 1. a) $\mathrm{N}_{2}(77 \mathrm{~K})$ and $\mathrm{CO}_{2}$ (195K) adsorption isotherms of FOS-1. Powder X-ray diffraction patterns of (b) FOS-1 (blue line) and (c) FOS-2 (green line). d) HRTEM image of FOS-1, showing its highly ordered structure; e) 2D-FFT pattern of the area contained in the square of panel $d$ ).

Details of the structure at the molecular level were highlighted by solid state NMR spectroscopy: a) $1 \mathrm{D}^{1} \mathrm{H},{ }^{19} \mathrm{~F},{ }^{13} \mathrm{C}$ and $2 \mathrm{D}{ }^{1} \mathrm{H}^{-13} \mathrm{C}$ Phase Modulated Lee-Goldburg homonuclear decoupling HETCOR MAS NMR spectra decoupled from ${ }^{1} \mathrm{H}$ or ${ }^{19} \mathrm{~F}$ of FOS-1 and FOS-2 demonstrated the molecular structure of the monomer unit; b) ${ }^{19} \mathrm{~F}$ MAS spectra showed a single resonance for both mono-fluoro and di-fluoro $p$-phenylene groups due to the regularity of the condensation; c) ${ }^{29} \mathrm{Si} \mathrm{CP}$ MAS NMR and quantitative ${ }^{29} \mathrm{Si}$ MAS NMR spectra revealed the massive conversion of the triethylethoxysilane moieties, in particular the $\mathrm{T}^{2}$ and $\mathrm{T}^{3}$ signals indicated that an efficient condensation process, and no C-Si bond cleavage occurred (Supporting Information). 
The motional behavior of the $p$-phenylene moieties in the mesoporous fluoro-organosilicas was established by ${ }^{13} \mathrm{C}$ spinlattice relaxation measurements that are sensitive to a motional regime in the frequency range of hundreds of megahertz. Figure 2 shows the measurement of ${ }^{13} \mathrm{C}$ spin-lattice relaxation rates as a function of temperature, from 253 to $383 \mathrm{~K}$. These data enable correlation times as well as apparent activation energies of the motional processes to be established. The most efficient relaxation is obtained when the motional frequencies match the Larmor frequency of the observed nuclei. ${ }^{8}$

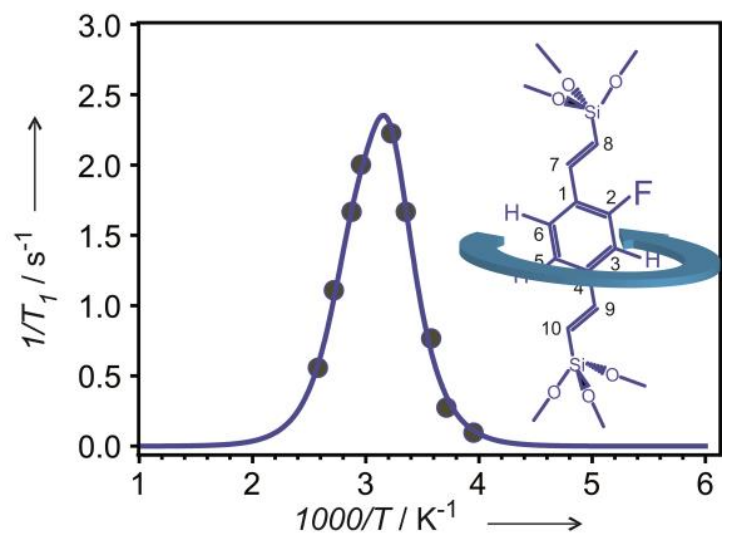

Figure 2. ${ }^{13} \mathrm{C}$ relaxation rates for $\mathrm{C}_{3}$ nucleus of FOS-1 versus the reciprocal of temperature as fitted by non linear mean-square analysis of Kubo-Tomita equation.

The ${ }^{13} \mathrm{C}$ relaxation rates at $75 \mathrm{MHz}$ for the $\mathrm{C}_{3}$ carbon atoms of FOS-1 show maximum values at $325 \mathrm{~K}$, consistent with fast molecular motions of the phenyl-ring moieties about their molecular rotation axes, with a mean correlation time $\tau_{c}$ as short as $2 \mathrm{~ns} .{ }^{9}$ Each aromatic ring possesses a lateral van der Waals steric hindrance of ca. $0.34 \mathrm{~nm}$ (each p-electron cloud accounts for $0.17 \mathrm{~nm}$ ) and the distance between adjacent axes of the diphenylene moieties imposed by the framework is ca. $0.44 \mathrm{~nm}$, as established by XRD. Consequently, there is much more space available than that of a closely packed face-to-face configuration, permitting rotational motion of dipolar $p$-phenylene groups. Indeed, correlation times of $10^{-9} \mathrm{~s}$ reflect a framework structure with unusual internal molecular mobility. Analyses of the temperature dependence of the relaxation rates yield an apparent activation energy of $9.6 \mathrm{kcalmol}^{-1}$, which is comparable or lower than the energy value reported in molecular crystals for a $180^{\circ}$ flip process of parasubstituted monofluoro-phenyl rings about their axes. ${ }^{3 b, c}$ The $180^{\circ}$ flip process was demonstrated by ${ }^{2} \mathrm{H}$ NMR of the non-fluorinated analogue compound tetradeutero-divinyl- $p$ phenylenesilica.

The presence of oscillating C-F dipoles in the frameworks allows the observation of the peculiar dielectric properties of the materials. The electric energy loss reaches its maximum at a temperature where the thermally activated reorientation rate equals the frequency of the external field. Thus, the maximum peak dissipation is a signature of thermally activated motion. To allow for easy identification of the dielectric contributions due to rotation of the fluorinated phenyl moiety, we compared the dielectric loss of both FOS-1 and FOS-2 materials to an analogue material where the phenyl rings lack fluorine substitution (Figure 3).
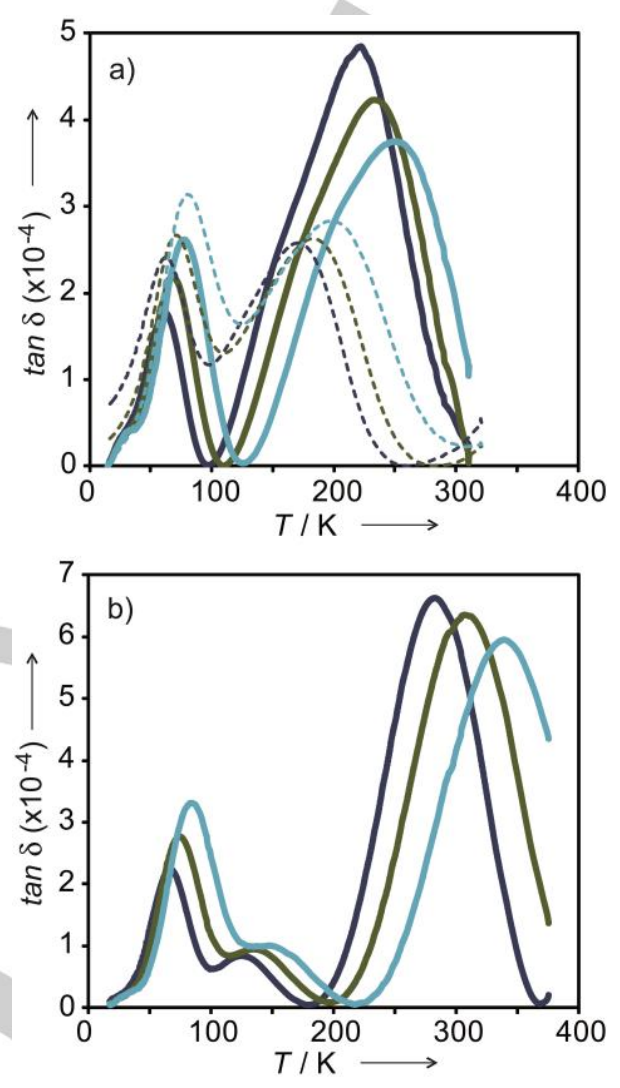

Figure 3. The dielectric loss (in the unit of loss tangent) at different temperatures of FOS-1 (a) and FOS-2 (b). Measurements are taken at different temperatures and at three different frequencies. Black: $140 \mathrm{~Hz}$, green: $1400 \mathrm{~Hz}$, and light blue: $14 \mathrm{kHz}$. Solid lines: FOS-1 and FOS-2. Dotted lines: non fluorinated mesoporous material.

Going from the lowest temperatures to the highest, the first loss structure occurs between $50 \mathrm{~K}$ and $100 \mathrm{~K}$ for the measurement frequencies reported. This dielectric loss peak is similar in all three materials, suggesting that it is not associated to the motion of $p$-phenylene rotors that bear a different number of fluorine atoms, but to the rotation of the common lateral vinyl moieties. The second set of loss peaks appears at higher temperatures. The un-fluorinated material shows dispersing losses between roughly $150 \mathrm{~K}$ and $225 \mathrm{~K}$, while FOS-1 losses are more intense, consistent with the polar structure containing the strong $\mathrm{C}-\mathrm{F}$ dipole, and occur at higher temperatures, between $175 \mathrm{~K}$ and $300 \mathrm{~K}$, in agreement with a higher barrier to motion. In FOS-2, the high-temperature loss peak is even stronger, consistent with a yet larger dipole moment, and appears at higher temperature, between $200 \mathrm{~K}$ and $325 \mathrm{~K}$, suggesting a higher rotational energy barrier.

The fitting of dispersion curves by a Debye model allows us to extract activation energies and attempt frequencies. For the lowest temperature peak, all three materials show activation energies as low as $3 \mathrm{kcal} / \mathrm{mol}$, with attempt frequencies $1 / \tau_{0}$ near $10^{-12} \mathrm{~s}^{-1}$. Instead, for phenyl rotations, the activation energies are centered at 12 and $14 \mathrm{kcal} / \mathrm{mol}$, in FOS1 and FOS-2 respectively, and attempt frequencies $1 / \tau_{0}$ of 
about $10^{-15} \mathrm{~s}^{-1}$ were measured. The rotational barrier measured for fluorinated phenyl ring rotation is in good agreement with the NMR results reported above, particularly given the significant differences in measurement frequencies and the resulting difference in temperature where the losses are maximized. Interestingly, the dipole reorientation in FOS-1 occurs at low temperature: in fact, the rotational frequency of $\mathrm{C}-\mathrm{F}$ dipoles, as fast as $1.4 \times 10^{4} \mathrm{~Hz}$, was achieved at $250 \mathrm{~K}$. This rate has been previously obtained only at much higher temperatures (room temperature ${ }^{3 b}$ and $317 \mathrm{~K}^{3 \mathrm{c}}$ ) in molecular crystals containing the same monofluoro- $p$-phenylene rotating moiety, vice versa, at room temperature our system is already in the Megahertz regime.

The above reported dielectric-loss observations, combined with NMR spectroscopy, have shown that the motional behavior can be thermally modulated. An alternative challenging approach for rotor-speed regulation is the direct intervention on the molecular moiety of the pore walls by a targeted chemical reaction. This was made possible by the sophisticated structure of the organic linkers that exhibited reactive unsaturation just on the rotor pivotal bonds, providing a handle for manipulation of the dipolar rotors. Thus, the matrix could be regarded as a reactive solid as the large porosity exposes massively its extended surface to the chemical action of a reactant. Interestingly, access to the reactive sites is provided by gaseous species even at extremely low pressures, e.g. by iodine vapors sublimed from solid elemental iodine at $50-60^{\circ} \mathrm{C}$ (vapor pressure of $4 \div 5$ torr), which could diffuse into the mesochannels (Figure 4).
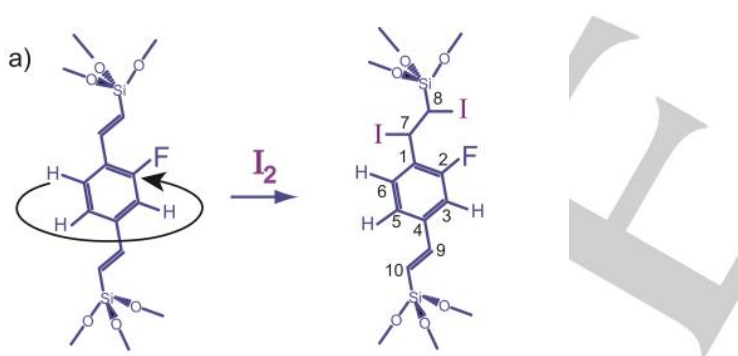

b)
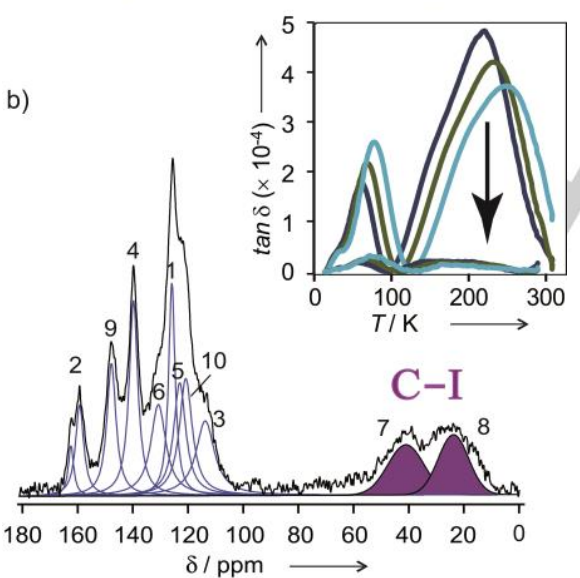

Figure 4. a) Schematic representation of $I_{2}$ addition on unsaturated double bonds of FOS-1. b) ${ }^{13} \mathrm{C}$ CP MAS NMR spectrum of FOS-1 after reaction with I. In the inset, the reduction of dielectric loss of iodine-reacted FOS-1, as compared to pristine FOS-1, is shown at the three distinct observation frequencies. Black: $140 \mathrm{~Hz}$, green: $1400 \mathrm{~Hz}$, and light blue: $14 \mathrm{kHz}$.
After a few hours, the $I_{2}$ addition reaction to the double bond vicinal to the aromatic fluorine atom (ortho substitution) of the divinyl-2-fluoro- $p$-phenylene moieties was largely accomplished. The occurrence of this reaction was recognized by solid-state NMR (Figure $4 \mathrm{~b}$ ) and the amount of reacted double bonds was quantified as $90 \%$ for unsaturation in the ortho position from fluorine atoms. Spin-lattice ${ }^{13} \mathrm{C}$ relaxation times became twenty times longer (from 0.6 to $13 \mathrm{~s}$ ) at room temperature, indicating that rotors are slowed down by the iodine addition and the fast motional frequency of $10^{8} \mathrm{~Hz}$ is achieved at temperatures $50 \mathrm{~K}$ higher than in the pristine matrix.

Dielectric measurements confirm this observation, in fact the strong dielectric loss peaks observed in FOS-1 below 300 $\mathrm{K}$ are drastically reduced (Figure 4). Reaction with iodine apparently suppressed fast rotational motion of fluoro- $p$ phenylene below room temperature, consistent with the shift of the dielectric loss peak to much higher temperatures and the increase in the rotational barrier height. The virtual absence of dispersive phenomena below room temperature in the reacted material indicates that the large part of the individual dipolar rotors can be tuned by a gas-solid reaction. Notably, the process demonstrates, unequivocally, that pores are fully accessible from the gas phase and can be explored, not only by the intrusive $\mathrm{N}_{2}$ and $\mathrm{CO}_{2}$ gases, as shown previously, but also by heavy and low-volatility species, such as molecular iodine.

In conclusion, a functional organization of dipolar molecular rotors was realized in mesoporous structures of organosilicas with aligned fluorinated $p$-phenylene moieties. Our design promoted the synthesis of fluorinated compounds, unprecedented in mesoporous organosilicas with molecular order. In fact, a single or a pair of fluorine atoms are inserted along one edge of each $p$-phenylene rotor, thus creating fastrotating dipoles stably anchored in the covalent framework. The arrangement of the dipolar rotors, aligned parallel to one another, generated periodic structures in which carbon-fluorine dipoles mounted on rotors easily reorient. The robust covalent framework was subjected to variable temperature dielectric and NMR relaxation measurements, which unequivocally established the rapid rotational frequency of the mobile elements $\left(\sim 10^{8} \mathrm{~Hz}\right.$ at $\left.325 \mathrm{~K}\right)$. The advantage of the materials being permanently porous, with open nanochannels accessible to gaseous species, permits the active manipulation of dipolar rotors through framework reactivity towards diffused-in chemicals. The present findings open up novel exciting perspectives for the use of such materials as selective sensors of chemical species, including radioactive elemental iodine and other vapours, as well as devices for drug delivery that work on the application of an electric field. In fact, the unique combination of multiple features, such as extended gas-solid interactivity, large storage capacity and stability of the covalent architecture with the rapidly rotating dipoles makes these materials promising prototypes for diversified applications spanning from gas capture and detection to nanomedicine. 


\section{Acknowledgements}

A.C. would like to thank PRIN 2011 and Cariplo Foundation 2012. K.Z. and C.T.R. gratefully acknowledge financial support from the US National Science Foundation Division of Materials Research, through Grant DMR-1409981.

Keywords: molecular rotors $\cdot$ porous materials $\cdot$ hybrid materials $\cdot$ NMR spectroscopy $\cdot$ dielectric spectroscopy

[1] a) G. S. Kottas, L. I. Clarke, D. Horinek, J. Michl, Chem. Rev. 2005 , 105, 1281-1376; b) C. S. Vogelsberg, M. A. Garcia-Garibay, Chem Soc. Rev. 2012, 41, 1892-1910; c) J. Neumann, K. E. Gottschalk, R. D. Astumian, ACS Nano 2012, 6, 5242-5248; d) A. Comotti, S. Bracco, T. Ben, S. Qiu, P. Sozzani, Angew. Chem. Int. Ed. 2014, 53, $1043-1047$

[2] a) Y. Zhang, W. Zhang, S.-H. Li, Q. Ye, H.-L. Cai, F. Deng, R.-G. Xiong, S. D. Huang, J. Am. Chem. Soc. 2012, 134, 11044-11049; b) T. Kutagawa, H. Koshinaka, D. Sato, S. Takeda, S. I. Noro, H. Takahashi, R. Kumai, Y. Tokura, T. Nakamura, Nat. Mater. 2009, 8, 342-347; c) W. Setaka, K. Yamaguchi, Proc. Natl. Acad. Sci. U.S.A. 2012, 109, 9271-9275

[3] a) R. D. Horansky, L. I. Clarke, E. B. Winston, J. C. Price, S. D. Karlen, P. D. Jarowski, R. Santillan, M. A. Garcia-Garibay, Phys.Rev. B 2006, 74, 054306; b) R. D. Horansky, L. I. Clarke, J. C. Price, T.-A. V. Khuong, P. D. Jarowski, M. A. Garcia-Garibay, Phys. Rev. B 2005 72, 014302; c) Q.-C. Zhang, F.-T. Wu, H.-M. Hao, H. Xu, H.-X. Zhao, L.-S. Long, R.-B. Huang, L.-S. Zheng, Angew. Chem. Int. Ed. 2013 52, 12602-12605; d) W. Setaka, K. Yamaguchi, J. Am. Chem. Soc. 2013, 135, 14560-14563.

[4] a) L. Kobr, K. Zhao, Y. Q. Shen, A. Comotti, S. Bracco, R. K. Shoemaker, P. Sozzani, N. A. Clark, J. C. Price, C. T. Rogers, J. Michl, J. Am. Chem. Soc. 2012, 134, 10122-10131; b) L. Kobr, K. Zhao, Y. Shen, K. Polivkova, R. K. Shoemaker, N. A. Clark, J. C.
Price, C. I. Rogers, J. Michl, J. Org. Chem. 2013, 78, 1768-1777; c) B. Rodriguez-Molina, M. E. Ochoa, M. Romero, S. I. Khan, N. Farfan, R. Santillan, M. A. Garcia-Garibay, Cryst. Growth Des. 2013, 13, 5107-5115; d) M. Brustolon, A. Barbon, M. Bortolus, A. L. Maniero, P. Sozzani, A. Comotti, R. Simonutti, J. Am. Chem. Soc. 2004, 126, 15512-15519; e) P. Sozzani, A. Comotti, S. Bracco, R. Simonutti, Angew. Chem. Int. Ed. 2004, 43, 2792-2797; f) A. Barbon, M. Bortolus, M. Brustolon, A. Comotti, A. L. Maniero, U. Segre, P. Sozzani, J. Phys. Chem. C 2003, 107, 3325-3331.

[5] a) E. B. Winston, P. J. Lowell, J. Vack, J. Chocholous, J. Michl, J. C. Price, Phys. Chem. Chem. Phys. 2008, 10, 5188-5191; b) S. Devautour-Vinot, G. Maurin, C. Serre, P. Horcajada, D. Paula da Cunha, V. Guillerm, E. de Souza Costa, F. Taulelle, C. Martineau, Chem. Mater. 2012, 24, 2168-2177.

[6] a) S. Inagaki, S. Guan, T. Ohsuna, O. Terasaki, Nature 2002, 416, 304-307; b) N. Mizoshita, T. Tani, S. Inagaki, Chem. Rev. Soc. 2012, 40,789-800. c) F. Hoffmann, M. Cornelius, J. Morell, M.. Froeba Angew. Chem. Int. Ed. 2006, 45, 3216-3251; d) M. Beretta, J. Morell, P. Sozzani, M. Froeba, Chem. Commun. 2010, 46, 2495-2497; e) C. S. Vogelsberg, S. Bracco, M. Beretta, A. Comotti, P. Sozzani, M. A. Garcia-Garibay, J. Phys. Chem. B 2012, 116, 1623-1632; f) S. Bracco, A. Comotti, P. Valsesia, B. F. Chmelka, P. Sozzani, Chem. Commun. 2008, 39, 4798-4800.

[7] a) N. Mizoshita, T. Tani, H. Shinokubo, S. Inagaki, Angew. Chem. Int. Ed. 2012, 51, 1156-1160; b) H. Takeda, M. Ohashi, Y. Goto, T. Ohsuna, T. Tani, S. Inagaki, Chem. Eur. J. 2014, 20, 9130-9136; c) A. Comotti, S. Bracco, P. Valsesia, L. Ferretti, P. Sozzani, J. Am. Chem. Soc. 2007, 129, 8566-8576; d) A. Comotti, S. Bracco, P. Valsesia, M. Beretta, P. Sozzani, Angew. Chemie Int. Ed. 2010, 49, 1760-1764.

[8] a) R. Kubo, K. Tomita, J. Phys. Soc. Jpn. 1954, 9, 888-919; b) A. Comotti, S. Bracco, A. Yamamoto, M. Beretta, T. Hirukawa, N. Tohnai, M. Miyata, P. Sozzani, J. Am. Chem. Soc. 2014, 136, 618621.

[9] F. A. Bovey, P. Mirau, in NMR of Polymers, Academic Press, San Diego, 1996 


\section{Table of Contents}

\section{COMMUNICATION}

Fluorinated molecular rotors were engineered in mesoporous hybrid organo-silicas with crystalline order in their walls. The rotor dynamics was established by MAS NMR and dielectric measurements, indicating $\tau_{c}$ as short as $10^{-9} \mathrm{~s}$ at $325 \mathrm{~K}$. The unusual combination of dipole reorientation with porosity enabled rotor speed regulation by chemicals entering the pores, such as iodine vapours which reacted with the pivotal double bonds and modulated the material dynamics.
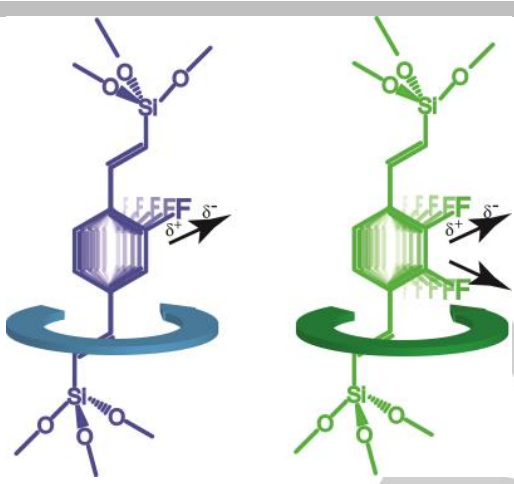

S. Bracco, M. Beretta, A. Cattaneo, A. Comotti, ${ }^{*}$ A. Falqui, K. Zhao, C. Rogers, P. Sozzani*

Page No. - Page No.

Dipolar Rotors Orderly Aligned in Mesoporous Fluoro-organosilica Architectures 